\title{
Algebraic Acceleration and Regularization of the Source Reconstruction Method with the Recompressed Adaptive Cross Approximation
}

\author{
Mahdi Kazempour and Levent Gürel \\ Bilkent University Computational Electromagnetics Research Center (BiLCEM), Ankara, TR-06800, Turkey \\ lgurel@gmail.com
}

\begin{abstract}
We present a compression algorithm to accelerate the solution of source reconstruction problems that are formulated with integral equations and defined on arbitrary threedimensional surfaces. This compression technique benefits from the adaptive cross approximation (ACA) algorithm in the first step. A further error-controllable recompression is applied after the ACA. The numerical results illustrate the efficiency and accuracy of the proposed method.
\end{abstract}

\section{INTRODUCTION}

One way to carry out antenna characterization and diagnosis in the far zone is to use the source reconstruction method (SRM) [1] as an alternative to the wave mode expansion (WME) technique [2]. The method obtains the equivalent distribution of the electric and magnetic currents on the reconstruction surface that radiate the same field as that of the antenna under test at the measurement surface. Unlike the WME technique, the SRM does not enforce any geometrical constraint on the reconstruction and measurement surfaces and can be applied on general 3-D surfaces [1].

The reconstruction surface is meshed using triangles on which Rao-Wilton-Glisson basis functions are defined. Furthermore, the measured field is sampled with a specific angular resolution. Thus, the desired discretization required for the numerical solution of the integral equation is achieved. Matching tangential components of the fields radiated from equivalent sources and the antenna under test on the measurement surface leads to a $2 L \times 2 N$ matrix equation, where $L$ is the number of sampling points on the measurement surface and $N$ is the number of unknowns. The matrix equation can be represented as

$$
\overline{\boldsymbol{Z}}_{(2 L \times 2 N)} \cdot \boldsymbol{a}_{(2 N \times 1)}=\boldsymbol{v}_{(2 L \times 1)},
$$

where the vector $\boldsymbol{a}$ contains the coefficients of the equivalent currents and $\boldsymbol{v}$ is the right-hand-side known vector that consists of the theta and phi components of the measured electric field, i.e., $E_{\theta}$ and $E_{\phi}$. Furthermore, in (1), the matrix $\bar{Z}_{(2 L \times 2 N)}$ consists of four submatrices of the size $L \times N$. Alternatively, (1) can be written as

$$
\left[\begin{array}{ll}
\overline{\boldsymbol{Z}}_{(L \times N)}^{E_{\theta}, \boldsymbol{J}} & \overline{\boldsymbol{Z}}_{(L \times N)}^{E_{\theta}, \boldsymbol{M}} \\
\overline{\boldsymbol{Z}}_{(L \times N)}^{E_{\phi}, \boldsymbol{J}} & \overline{\boldsymbol{Z}}_{(L \times N)}^{E_{\phi}, \boldsymbol{M}}
\end{array}\right] \cdot\left[\begin{array}{c}
\boldsymbol{a}_{J(N \times 1)} \\
\boldsymbol{a}_{M(N \times 1)}
\end{array}\right]=\left[\begin{array}{c}
\boldsymbol{v}_{E_{\theta},(L \times 1)} \\
\boldsymbol{v}_{E_{\phi},(L \times 1)}
\end{array}\right],
$$

where $\overline{\boldsymbol{Z}}^{E_{X}, \boldsymbol{J}}$ is a submatrix relating the $\boldsymbol{a}_{J}$ coefficients with the $X$ component of the electric field and $\bar{Z}^{E_{X}, M}$ relating the $\boldsymbol{a}_{M}$ coefficients to the $X$ component of the electric field on the measurement surface $(X \in\{\theta, \phi\})$ [1]. For the leastsquares solution of the matrix equation in (2), we perform the transformation

$$
\bar{Z} \cdot \boldsymbol{a}=\boldsymbol{v} \rightarrow \overline{\boldsymbol{Z}}^{H} \cdot \overline{\boldsymbol{Z}} \cdot \boldsymbol{a}=\overline{\boldsymbol{Z}}^{H} \cdot \boldsymbol{v},
$$

with $\overline{\boldsymbol{Z}}^{H}=\left\{\overline{\boldsymbol{Z}}^{T}\right\}^{\star}$. In (3), the superscript " $H$ " stands for the transpose and complex conjugate operation and $\overline{\boldsymbol{Z}}^{H} \cdot \overline{\boldsymbol{Z}}$ is a Hermitian matrix. The resulting matrix equation in (3) is ill conditioned and requires an appropriate regularization. To this end, a common technique is to use the singular value decomposition (SVD) method, which leads to a high-quality solution [3].

The iterative solution of (1) requires $\mathcal{O}(L N)$ operations, which becomes a bottleneck for problems with large $L$ and $N$. The situation worsens when SVD is applied for regularization with a computational cost of $\mathcal{O}\left(N^{2} \max (N, L)\right)$. Several fast methods are applied to reduce the computational cost of the SRM, such as techniques based on the fast multipole method, its multilevel version [4], and adaptive cross approximation (ACA) [5], [6]. In this work, we incorporate the recompressed ACA (RACA) [7] to reduce the memory usage and solution time of the SRM. A major advantage of RACA is that it is purely algebraic and, unlike SVD, it does not depend on the full knowledge of all matrix elements. Using RACA, we can compute SVD with low computational cost. Section II outlines the implementation details, Section III provides the numerical and we conclude in Section IV.

\section{IMPLEMENTATION}

In the first step, we will apply ACA on the $\bar{Z}$ matrix in (1) to obtain

$$
\overline{\boldsymbol{Z}}_{(2 L \times 2 N)}=\overline{\boldsymbol{U}}_{(2 L \times k)} \cdot \overline{\boldsymbol{V}}_{(k \times 2 N)}^{H},
$$

where $\overline{\boldsymbol{U}}$ and $\overline{\boldsymbol{V}}$ are complex matrices of rank $k$. By incorporating the QR decomposition and computing the SVD, as shown in Algorithm 1, the matrix equation in (3) becomes

$$
\begin{array}{r}
\overline{\boldsymbol{V}}_{\left(2 N \times k^{\prime}\right)} \cdot \overline{\boldsymbol{U}}_{\left(k^{\prime} \times 2 L\right)}^{H} \cdot \overline{\boldsymbol{U}}_{\left(2 L \times k^{\prime}\right)} \cdot \overline{\boldsymbol{V}}_{\left(k^{\prime} \times 2 N\right)}^{H} \cdot \boldsymbol{a}_{(2 N \times 1)} \\
=\overline{\boldsymbol{V}}_{\left(2 N \times k^{\prime}\right)} \cdot \overline{\boldsymbol{U}}_{\left(k^{\prime} \times 2 L\right)}^{H} \cdot \boldsymbol{v}_{(2 L \times 1)},
\end{array}
$$

where $k^{\prime}$ is the effective rank. Once (5) is obtained, the required matrix-vector multiplications in the iterative solver 
are efficiently computed with $2 k^{\prime}(N+L)$ operations. The proposed method also benefits from reduced memory consumption. The computational complexity of the provided compression technique is less than the SVD [7]. Furthermore, the ill-conditioned matrix equation in (3) is regularized by keeping the first $k^{\prime}$ significant singular values.

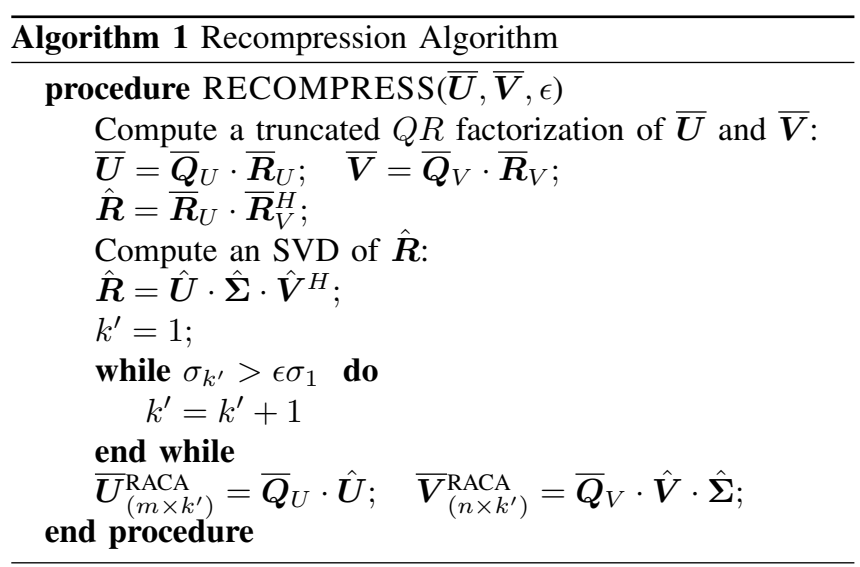

\section{NUMERICAL RESULTS}

To verify the proposed method, we consider the following scenario: The operating frequency is $1 \mathrm{GHz}$. A $4 \times 1$-element array of $z$-oriented dipoles with half-wavelength separation are placed on the $z$-axis, as shown in Fig. 1 . The array is enclosed by a sphere of radius $1 \lambda$ and the equivalent sources will be reconstructed on this sphere. The measurement surface is a sphere of radius $2.5 \lambda$. The theta component of the electric-field patterns on a path with $r=5 \lambda, \phi=0^{\circ}$, and $0^{\circ} \leq \theta \leq 180^{\circ}$ are compared and a good agreement is observed (Fig. 2). The relative error between the fields from equivalent sources and original sources is calculated via

$$
\text { Relative Error }=100 \times \frac{\left|E_{\theta, \text { orig }}-E_{\theta, \text { equiv }}\right|}{\left|E_{\theta, \text { orig }}\right|},
$$

and is illustrated by a green line in Fig. 2. In these simulations, the convergence residual of the BiCGSTAB iterative solver is set to $10^{-4}$ and the threshold for RACA is set to $\epsilon=10^{-3}$. The total reconstruction time using the ordinary method is 1572 seconds, whereas RACA reduces the CPU time to 184 seconds. With RACA, a $91 \%$ reduction in memory consumption and a speed-up of factor 8.5 are obtained. Ordinary ACA (without recompression) would lead to $78 \%$ memory usage reduction and a speed-up of factor 4.1. Hence, we can conclude that RACA outperforms ordinary ACA.

\section{CONCLUSION}

We propose an error-controllable matrix compression technique to reduce the computational cost of the SRM and to obtain a low-cost regularization. With the proposed technique, a significant reduction in memory (up to 91\%) is achieved compared to the ordinary SRM. The SRM results agree well with the reference solutions, testifying to the accuracy of the method.

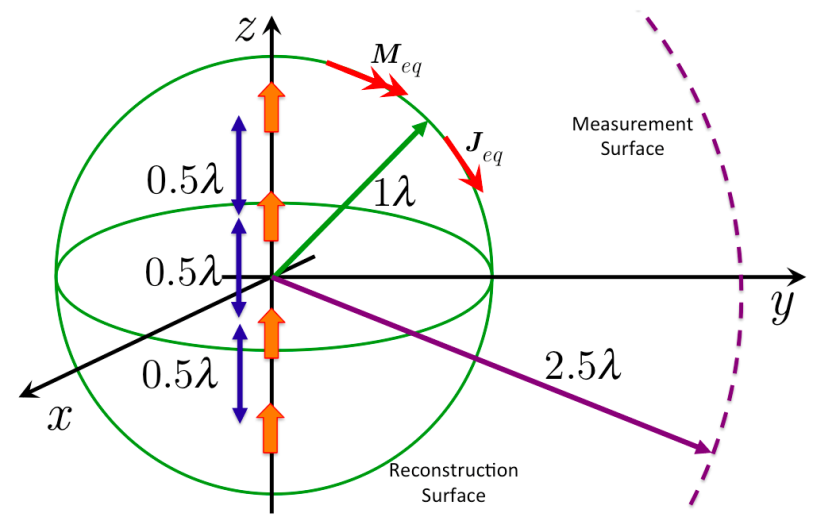

Fig. 1. Original $4 \times 1$-element array of dipoles with $0.5 \lambda$ separation (orange arrows). The green sphere indicates the reconstruction surface.

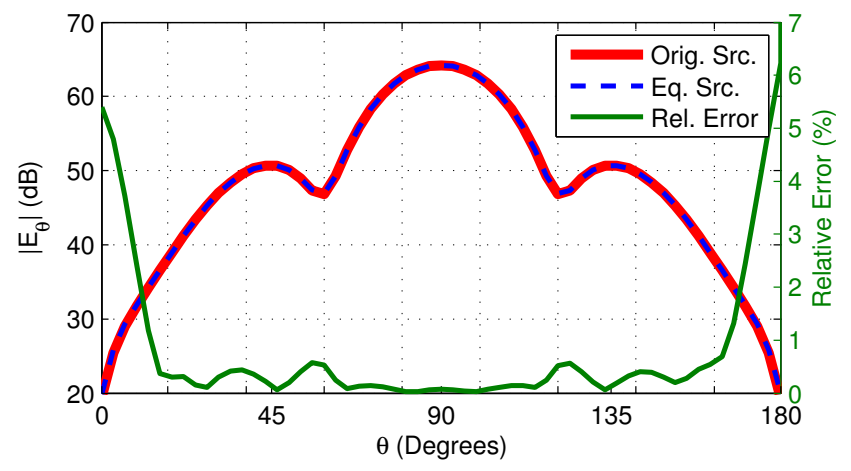

Fig. 2. Comparison of the fields radiated from the original and equivalent currents on a path with $r=5 \lambda, \phi=0^{\circ}$, and $0^{\circ} \leq \theta \leq 180^{\circ}$. The solid red curve represents the magnitude of the theta component of the original electric field. The dashed blue curve indicates $\left|E_{\theta}\right|$ that is radiated from the equivalent currents. The green curve illustrates the percentage of the relative error.

\section{ACKNOWLEDGEMENT}

This work was supported by the Scientific and Technical Research Council of Turkey (TUBITAK) under Research Grant 111E203, by Schlumberger-Doll Research (SDR), and by contracts from ASELSAN, Turkish Aerospace Industries (TAI), and the Undersecretariat for Defense Industries (SSM).

\section{REFERENCES}

[1] Y. Alvarez, F. Las-Heras, M. R. Pino, and T. K. Sarkar, "An improved super-resolution sources reconstruction method," IEEE Trans. Instrum. Meas., vol. 58, no. 11, pp. 3855-3866, 2009.

[2] A. D. Yaghjian, "An overview of near-field antenna measurements," IEEE Trans. Antennas Propag., vol. 34, no. 1, pp. 30-45, 1986.

[3] G. H. Golub and C. F. Van Loan, Matrix Computations, Baltimore, MD: The John Hopkins University Press, 1996.

[4] T. Eibert and C. Schmidt, "Multilevel fast multipole accelerated inverse equivalent current method employing Rao-Wilton-Glisson discretization of electric and magnetic surface currents," IEEE Trans. Antennas Propag., vol. 57, no. 4, pp. 1178-1185, 2009.

[5] Y. Alvarez, F. Las-Heras, and M. R. Pino, "Application of the adaptive cross approximation algorithm to the sources reconstruction method," $3 \mathrm{rd}$ Eur. Conf. Antennas Propag. (EuCAP'09), 2009.

[6] M. Bebendorf, "Approximation of boundary element matrices," Numer. Math., vol. 86, no. 4, pp. 565-589, 2000.

[7] M. Bebendorf, Hierarchical Matrices: A Means to Efficiently Solve Elliptic Boundary Value Problems, Berlin: Springer-Verlag, 2008. 